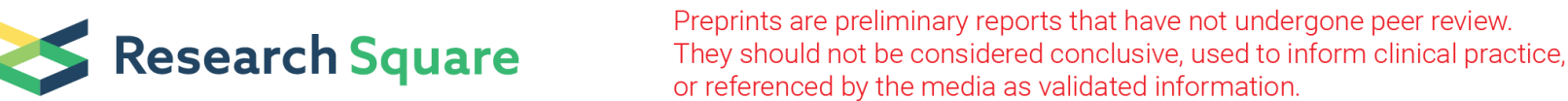

\section{Evaluation of COVID-19 Pandemic in Six Asia- Pacific Countries using Data-Driven and Machine Learning Method Based on the Current Management and Interventions}

\section{Shuang Ao}

Beijing Engineering Research Center of Food Environment and Public Health, Minzu University of China

Wenbo Huang

Beijing Information Engineer College: Beijing Information Science and Technology University

Dan Han

Minzu University of China

Yuming Liu

Minzu University of China

Shuang Liu

Minzu University of China

yaojiang huang ( $\nabla$ yaojiang@muc.edu.cn )

Minzu University of China https://orcid.org/0000-0001-5409-5605

Research article

Keywords: COVID-19, Pandemic, Evaluate, Machine Learning, Asia-Pacific

Posted Date: February 23rd, 2021

DOI: https://doi.org/10.21203/rs.3.rs-251813/v1

License: (c) (i) This work is licensed under a Creative Commons Attribution 4.0 International License.

Read Full License 


\section{Abstract}

Background South-east Asia and Western Pacific countries have large populations and underreporting of Covid19, which pose challenges to the large-scale response.

Methods Data-driven methods are used to evaluate the Government or society's interventions and the situation of the COVID-19 pandemic, and machine learning method are used to forecast the trend of COVID-19 pandemic based on the current management and interventions.

Results The results show that. India received low government response index scores in February, and the number of confirmed cases and active cases in September became quite high with large stock and the overall growth rate is higher than 1. The number of daily confirmed cases in Bangladesh, Japan and Philippines is low and on the decline, it is rising in Malaysia and Indonesia. The number of active cases in Bangladesh, Japan, India and Bangladesh has begun to decline, Malaysia and Indonesia is no sign of decline. Bangladesh, Japan and Philippines will be flat or moderating, while Malaysia and Indonesia will still have no slowdown momentum and the situation will be severe.

Conclusions The results show that the existing management and interventions responses are effective, although they have room for improvement, and Malaysia and Indonesia need to be improved and strengthened.

\section{Introduction}

Real-time data show that the confirmed cases of COVID-19 are growing like crazy in most countries of the world[1] [2]. Countries or regions of the world have adopted different social, economic, health and environmental measures to intervene in the epidemic, there is with no vaccine or drug are available, which interventions focus on social distancing and movement restrictions, contact tracing, isolation of suspected and confirmed cases, Lockdowns and public health measures [3] [4,5]. However, the COVID-19 transmission continues to spread in many countries or regions and has not achieve control. It is still a major public health crisis and in the world】although some countries, like South Korea and China, are gradually lifting these measures after achieving effective control over transmission [1] [6] [3]. However, long or intermittent governmental interventions and responses may be required to reduce the possibility of infection recurrence.

The countries of South-east Asia and Western Pacific are among the first to experience COVID-19 outbreaks with huge populations and relatively scarce public health resources, so the risk of pandemics of infectious diseases is relatively high. At the same time, high levels misdiagnosis and of under-reporting make a challenges for government to large-scale responses [7] [8]. Therefore, it is necessary to conduct correct evaluation, modeling and forecasting studies on epidemic data to estimate the potential impact of interventions during the uncertain phase of the epidemic and to provide reference and guidance for the next step of epidemic prevention and control, which is critical if local transmission continues to increase [9] [10] [11] [5]. 
Driven by a large amount of data from our life and health record, through interdisciplinary exploration in data science, statistics, network science, physics and epidemiology, a simple mathematical models describing the essence of the spread of epidemics can be generated and used to simulate and fit data with a number of appropriate parameters and make informed forecast [12] [13] [3] [14]. A large amount of evidence in recent decades shows that mathematical modeling and prediction have made great progress in better understanding the spread and transmission dynamics of infectious diseases, and played a key role in finding the optimal containment strategies in the spread of epidemics [15] [16] [17] [18] [19].

In this study, under the existing interventions and responses, we conducted data-driven comprehensive analysis and machine learning method to evaluate and forecast the COVID-19 transmission in countries with top three confirmed cases in Western Pacific and South-east Asia as of Jan 30, 2021. It aims to assess the interventions and responses in these countries and the status of the pandemic, establish a national spatial model of the spread of COVID-19 to estimate the time distribution and development trend of COVID-19 in each country. We would be pleased if our work could contribute to further research and applications that could help the effective interventions of the COVID-19 epidemic.

\section{Materials And Methods}

\subsection{Data Source}

The three countries with the largest number of cumulative confirmed COVID-19 cases in South-east Asia and western pacific on Jan 30, 2021 were selected as study subjects. Data Source: 2019 Novel Coronavirus COVID-19 (2019-nCoV) Data Repository by Johns Hopkins CSSE (https://github.com/CSSEGISandData/COVID-19/tree/master/csse_covid_19_data), Novel Corona Virus 2019 Dataset (https://www.kaggle.com/sudalairajkumar/novel-corona-virus-2019-dataset), The World Bank Total Population Data (https://data.worldbank.org/indicator/SP.POP.TOTL), Oxford COVID-19 Government Response Tracker (OxCGRT) (Oxford, 2020) and ACAPS COVID-19 Government Measures Dataset [5].

\subsection{Governmental interventions and responses}

The interventions and responses were analyzed as response stringency Index through R "tidycovid19" package [25]. The OxCGRT project composite measure of nine of metrics (stay-at-home requirements, restrictions on public gatherings, school closures, public information campaigns, workplace closures, closures of public transport, cancellation of public events, international travel controls and restrictions on internal movements) to calculate a government stringency Index. The index score of the nine metrics between 0 and 100 on any given day.

\subsection{Assessment of COVID-19 pandemic based on the current interventions}

The overall situation, daily changes and growth rates, total confirmed cases per million people, total deaths per million people and case fatality rate (CFR) of the ongoing COVID-19 pandemic based on the 
current interventions and responses in each country were compute using "EpiModel” package and "COVID19.Analytics" package in R [26].

\subsection{The trend of COVID-19 pandemic based on the current policy environment}

Machine Learning method[27] using to forecasting the trend of COVID-19 pandemic based on the existing interventions and responses in Japan, Philippines, Malaysia, India, Bangladesh and Indonesia and Generating a 365 day ahead forecast of confirmed cases of COVID-19 with 95\% prediction interval (PI). The algorithm and formula are:

$$
y(t)=g(t)+s(t)+h(t)+\epsilon_{t} \quad \text { and } \quad g(t)=(k+\boldsymbol{a}(t) \delta) \cdot t+\left(m+\boldsymbol{a}(t)^{T} \gamma\right) .
$$

A basic model was established with no tweaking of season-related parameters and additional regression elements. Machine Learning method were used to model and predict the number of active cases in each country. The Machine Learning method will assign each day in future a predicted value which it names yhat, Yhat_lower and Yhat_upper are the upper and lower yhat of the prediction (based on 95\% prediction Interval).

\section{Results}

As of Jan 30, 2021, the three countries with the largest number of cumulative confirmed COVID-19 cases in South-east Asia and western pacific include Japan, the Philippines, Malaysia, India, Bangladesh and Indonesia, which we selected as study subjects.

\subsection{Government interventions and responses}

The interventions and responses were evaluated as Response Stringency Index through R package "Tidycovid19" to summarize the severity of the interventions and responses, a higher index score indicates a government response stricter. The median interventions and responses index is 38.89 for Japan, 80.09 for Bangladesh, 73.61 for India, 68.98 for the Philippines, 59.72 for Indonesia and 57.41 for Malaysia (Figure $1 \mathrm{a}, \mathrm{b}$ ). Total confirmed cases per million people of the ongoing COVID-19 pandemic is 3084.89 (Japan), 3249.38 (Bangladesh), 7795.35 (India), 4796.61 (the Philippines), 3942.31 (Indonesia) and 6641.51 (Malaysia) (Figure 1 b Figure S1). In general, the number of confirmed cases per million decreased with the increase of the government responses stringency index (Figure 1. a, b). India received low government response index scores in February, and as a result, the number of cases was already very high in September. It can be seen that the government's early anti-epidemic measures were very important.

\subsection{Assessment of COVID-19 pandemic based on the current interventions}

\subsubsection{The overall situation of the epidemic}


The total number of confirmed cases, death, recovered and active cases show in Figure2 in Jan 30, 2021. Japan had 387499 confirmed COVID-19 cases, 325436 recovered, 5688 deaths and 56375 active cases, indicating a downward trend in the number of active cases (Figure 2a). In the Philippines, 523516 confirmed cases, 475904 recovered, 10669 deaths and 36943 active cases have been confirmed with a wave (Figure 2b). The number of confirmed COVID-19 cases was 209661, recovered patients was 161527 , death was 746 , and the number of active cases was 47388 with a going up in waves in Malaysia (Figure 2c). In India, there were 10.74617 M confirmed cases, $10.42311 \mathrm{M}$ recovered, 154274 death and 168791active cases, indicating that the number of active cases showed a decreasing trend (Figure $2 \mathrm{~d}$ ). 534770 confirmed cases, 479297 recovered, 8111deaths and 47362 active cases have been reported In Bangladesh, with the number of active cases showed a decreasing trend (Figure 2e). Indonesia had $1.066313 \mathrm{M}$ confirmed cases, 862502 recovered, 29728 deaths, and 174083 active cases. The number of active cases and confirmed cases is still on the rise in Indonesia (Figure 2f).

The Figure 3 show the total number of cases for six countries with a confidence band based on moving average and overall growth rate. Plot the number of cases as a function of time for the given locations and type of categories to generate different fits to match the data in a log-scale scatter and a linear scale bar plot. The overall growth rate is closer to 1 in Japan and Malaysia (Figure 3a and c), indicating its almost stopped growth, while in Philippines and Indonesia is always higher than 1 (Figure $3 \mathrm{~b}$ and $\mathrm{f}$ ), the number of cases in these countries is constantly increasing, especially in India and Bangladesh (Im-exp $\mathrm{GR}=1.04$ ), where the growth rate is much higher than 1 (Figure $3 \mathrm{~d}$ and $\mathrm{e}$ ), indicating its close to the exponential growth.

\subsubsection{Daily changes and growth rates}

Daily confirmed cases changes were computed that display two scatter plots per country with the number of changes, both in log-scale (right vertical axis) and linear scale (left vertical axis) combined in upper panel. The growth rate between two consecutive dates in confirmed cases that display a bar plot as a function of time in bottom panel (Figure $4 \mathrm{a}-\mathrm{f}$ ). Display multiple plots in one figure in a mosaic type layout and generate a heatmaps comparing the daily confirmed cases changes per day in the different country (Figure $4 \mathrm{~g}$ ).

The results showed that Japan, Philippines, India and Bangladesh had the highest number of daily confirmed cases on January 8, 2021(7863 people), August 4, 2020 (6263 people), September 10, 2020 (96511 people) and July 2 (4019 people), respectively (Figure 4 a, b, d e and, g). After that, the number of daily confirmed cases in three countries began to decline, the daily decline in the number of confirmed cases in Japan and Philippines has not been large and is therefore likely to rise again. However, the number of confirmed cases and the growth rate in a single day are still increasing in the Malaysia (confirmed cases, 5728/growth rate, 1.000524), and Indonesia (confirmed cases, 14518/growth rate, 1.051877), showing no sign of reaching the peak (Figure $4 \mathrm{c}, \mathrm{f}$ and $\mathrm{g}$ ). , the number of confirmed cases increased by more than 13000 in a single day in Indonesia and India, which situation is still not encouraging. 
3.2.3 Total confirmed cases, deaths per million people and CFR of the COVID-19 pandemic

The total confirmed cases, deaths per million people, CFR vs. tests per confirmed cases and tests per capita of the ongoing COVID-19 pandemic were calculated. Japan had the lowest and India had the highest in the number of confirmed COVID-19 cases per million India has the highest total number of

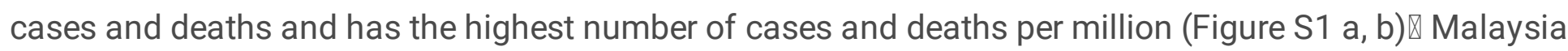
has the lowest and Indonesia has the highest in CFR of the ongoing COVID-19 pandemic. However, Indonesia has the lowest and Malaysia has the highest in tests per confirmed case of the ongoing COVID19 pandemic (Figure S1 c, d).

\subsection{The trend of COVID-19 pandemic}

COVID-19 pandemic were forecasted using Machine Learning method to project the spread of the SARSCoV-2 based on the interventions and responses in the next 365 days by a basic model with no tweaking of season-related parameters and additional regression elements and prediction interval of $95 \%$.

The Machine Learning method will assign each day in future a predicted value which is yhat, Yhat_lower and Yhat_upper. Under existing government or society's intervention and prevention actions in each country (Figure 1), machine learning forecasts show that by Jan 29, 2022, Bangladesh(yhat: 1.119778e+06, PI: 191530-2.074636e+06) will be flat or even stop growing in the number of total confirmed cases, Japan(yhat: 1.346271e+06, PI: 877532-1.832912e+06), Philippines(yhat: 1.046895e+06, Pl: 195077-1.954894e+06) and India (yhat: 2.179884e+07, PI: 3.330251e+064.030408e+07) will be flat and moderating, while Malaysia (yhat: 731846, PI: 527689-936022) and Indonesia (yhat: 3.386939e+06, PI: 2.084572e+06-4.620615e+06) will still have no slowdown momentum, will continue to grow and the situation will be severe (Figure 5).

Under existing interventions and responses (such as social distance conditions) in each country (Figure 1), Philippines, Bangladesh, India (on September, 2020) and Japan (on January, 2021) has peaked and The number of active cases are decreasing, however the number of active cases in the Malaysia and Indonesia is far from reaching its peak and is continuing to rise (Figure $5 \mathrm{a}-\mathrm{f}$ ).

\section{Discussion}

The interventions and the overall situation of the COVID-19 pandemic were evaluated by data-driven methods. India received low government response index scores in February, and the number of confirmed cases in September became quite high (Figure 1b, Figure $2 \mathrm{~d}$ ), indicating that the early epidemic interventions and responses are very important. The median of Governmental response Stringency Index is 80.09 in Bangladesh with the most stringent interventions and responses (Figure 1a and b), India and the Philippines are also high, have shown a trend of slowdown in confirmed cases (Figure 5) and decline in daily confirmed case (Figure 4). In Indonesia and Malaysia, The median of Governmental response Stringency Index is relatively low, and the number of total confirmed cases (Figure 2, 3, 5) and daily confirmed cases are on the rise so far (Figure $4 \mathrm{c}$ and $\mathrm{f}$ ). The number of active cases in Bangladesh, 
Japan and Philippines showed increasing trend. The number of active cases in India showed a trend of slowing down (168791, Figure 2d, Figure 5d) with large stock and the overall growth rate is higher than 1. The number of active cases in Malaysia and Indonesia showed a large stock and an increasing trend (47388 and 174083, Figure 2c and f, Figure $5 \mathrm{c}$ and $\mathrm{f}$ ) with the overall growth rate is always higher than 1 (Figure $3 \mathrm{c}$ and $\mathrm{f}$ ). The high number of active cases, growth rate well above 1 and the rising trend mean that the number of confirmed cases will continue to rise, and the situation will become more and more serious before the epidemic reaches its peak and develops for a long time.

Machine learning forecasts under current interventions and responses show that the Philippines, India, Japan and Bangladesh will be flat and moderating, while Indonesia and Malaysia will still have no slowdown momentum and the situation will be severe (Figure $5 \mathrm{a}-\mathrm{f}$ ). The number of active cases in the Philippines, India, Bangladesh and Japan shows a downward trend after peaking in September, 2020 and January, 2021, respectively. The number of active cases in Malaysia and Indonesia is no sign of decline and the situation will be severe (Figure 5 a-f, Figure 4 a-f).

These findings show that although the total number of confirmed cases of COVID-19 is still increasing, the existing interventions and responses are still effective, because in general, the number of confirmed cases per million decreased with the increase of the government response stringency index. Interestingly, the higher the Tests per confirmed case is, the smaller the CFR will be. The number of daily confirmed cases and active cases of COVID-19 in Malaysia and Indonesia is no sign of decline, indicate the interventions and responses of these three countries still have room for improvement or do better.

Our study could have some limitations. First, the daily and total number of confirmed cases is not the actual daily number of infections and the number of total cases, because not all infected persons are tested, especially in countries that do not actively track, test and isolate suspected and close contacts, the number of confirmed cases and active cases may be much lower than true number of infections [21] [22] [23]. Only when a country or region can effectively isolate all infected and suspected persons, track, detect and isolate all close contacts, have strong detection capabilities and carry out large-scale screening, then the daily and total number of confirmed cases close to the true number of infections.

Second, our assessment and projection are based on the status quo that is the existing level of government Response, social distance, wearing masks and so on, but the status quo is unsustainable because all kinds of environment and conditions such as government response, social distancing, whether to wear masks are subject to change, and the country and society may need to gradually open up [5] [4], so the predicted results will also change accordingly. The peak of daily growth in some countries does not mean that they are in the clear, unless more stringent government measures, contact tracing and isolation, social distancing, and universal wearing of masks are maintained or even implemented.

Finally, the evaluation of the COVID-19 at this stage is very challenging based on the above reasons and the situation of the global epidemic, and the forecast of the cases numbers and trend of the COVID-19 must be carefully interpreted [24]. However, we are convinced that the results on cumulative case changes and machine learning predictions are much more reliable, because these are based on the shape of the 
COVID-19 epidemic curve to make machine learning predictions and not only based on the number of cases [17] [3] [15] [18]. We believe that widespread use of the vaccine could speed up the recovery, and our projections is useful to the effective interventions and taken against of the COVID-19 epidemic.

\section{Conclusions}

Data-driven method were used to assessment the status of the pandemic and machine learning method were used to forecast the COVID-19 pandemic in Asia-Pacific countries under the existing policy environment. This findings show that although the total number of confirmed cases of COVID-19 is still increasing, the number of daily confirmed cases and active cases of COVID-19 in Japan, the Philippines, India and Bangladesh has begun to decline, while active cases and daily confirmed cases is no peak in Malaysia and Indonesia, indicate that the existing interventions and responses in Japan, the Philippines, India and Bangladesh are effective, while Malaysia and Indonesia need to be improved and strengthened. This study will be helpful in the response to COVID-19 pandemic.

\section{Abbreviations}

COVID-19: Coronavirus disease 2019, WHO: The World Health Organization, OxCGRT: Oxford COVID-19 Government Response Tracker, PI: 95\% prediction Interval, CFR: case fatality rate, yhat: each day in future a predicted value.

\section{Declarations}

\section{Funding}

Double First Class University Plan (MOE, China Ministry of Education) and Beijing Municipal Science and Technology Commission.

\section{Conflicts of interest/Competing interests}

All authors declare no competing interests.

\section{Ethics approval}

Not applicable

\section{Consent to participate}

Not applicable 


\section{Consent for publication}

Not applicable

\section{Availability of data and material}

Data is available from the corresponding author upon reasonable request.

\section{Code availability}

Code is available from the corresponding author upon reasonable request.

\section{Authors' contributions}

Yaojiang Huang: Conceptualization, Methodology, Code and Software, Validation, Formal analysis, Writing - review \& editing, Data interpretation, Supervision. Shuang Ao: Investigation, Methodology, Code and Software, Writing-original draft, Writing - review \& editing, Visualization. Wenbo Huang: Investigation, Methodology, Code and Software, Writing-original draft, Writing - review \& editing, Visualization, Data interpretation. Dan Han: Investigation, Methodology, Writing-original draft, Writing - review \& editing, Validation, Writing - original draft, Writing - review \& editing. Yuming Liu: Validation, Writing - review \& editing, Visualization. Shuang Liu: Validation, Formal analysis, Writing - review \& editing.

\section{References}

1. WHO. Coronavirus Disease 2019 (COVID-19): Situation Report-193 2020. Availabe online: https://www.who.int/docs/default-source/coronaviruse/situation-reports/20200731-covid-19-sitrep193.pdf?sfvrsn=42a0221d_4 (accessed on

2. JHU, T.C.f.S.S.a.E.C.a. COVID-19 Map - Johns Hopkins Coronavirus Resource Center. Availabe online: https://coronavirus.jhu.edu/map.html (accessed on

3. Jewell, N.P.; Lewnard, J.A.; Jewell, B.L. Predictive mathematical models of the COVID-19 pandemic: Underlying principles and value of projections. Jama 2020, 323, 1893-1894, doi:10.1001/jama.2020.6585.

4. Oxford. Oxford COVID-19 Government Response Tracker (OxCGRT) Availabe online: https://covidtracker.bsg.ox.ac.uk/ (accessed on

5. ACAPS. ACAPS COVID-19 Government Measures Dataset 2020.

6. WHO. Coronavirus Disease 2019 (COVID-19): Situation Report-204 2020. Availabe online: https://www.who.int/docs/default-source/coronaviruse/situation-reports/20200811-covid-19-sitrep204.pdf?sfvrsn=1f4383dd_2 (accessed on 
7. Din, M.; Asghar, M.; Ali, M. COVID-19 and dengue coepidemics: A double trouble for overburdened health systems in developing countries. Journal of medical virology 2020, 10.1002/jmv.26348, doi:10.1002/jmv.26348.

8. Krishnan, L.; Ogunwole, S.M.; Cooper, L.A. Historical Insights on Coronavirus Disease 2019 (COVID19), the 1918 Influenza Pandemic, and Racial Disparities: Illuminating a Path Forward. Annals of Internal Medicine 2020, 10.7326/M20-2223, doi:10.7326/M20-2223.

9. Perc, M.; Gorišek Miksić, N.; Slavinec, M.; Stožer, A. Forecasting Covid-19. Frontiers in Physics 2020, 8, 127, doi:10.3389/fphy.2020.00127.

10. Anastassopoulou, C.; Russo, L.; Tsakris, A.; Siettos, C. Data-based analysis, modelling and forecasting of the COVID-19 outbreak. PloS one 2020, 15, e0230405, doi:10.1371/journal.pone.0230405.

11. Grasselli, G.; Pesenti, A.; Cecconi, M. Critical care utilization for the COVID-19 outbreak in Lombardy, Italy: early experience and forecast during an emergency response. Jama 2020, 323, 1545-1546, doi:10.1001/jama.2020.4031.

12. Cássaro, F.A.; Pires, L.F. Can we predict the occurrence of COVID-19 cases? Considerations using a simple model of growth. Science of the Total Environment 2020, 10.1016/j.scitotenv.2020.138834, 138834, doi:10.1016/j.scitotenv.2020.138834.

13. Salathe, M.; Bengtsson, L.; Bodnar, T.J.; Brewer, D.D.; Brownstein, J.S.; Buckee, C.; Campbell, E.M.; Cattuto, C.; Khandelwal, S.; Mabry, P.L. Digital epidemiology. PLoS Comput Biol 2012, 8, e1002616, doi:10.1371/journal.pcbi.1002616.

14. Pastor-Satorras, R.; Castellano, C.; Van Mieghem, P.; Vespignani, A. Epidemic processes in complex networks. Reviews of modern physics 2015, 87, 925, doi:10.1103/RevModPhys.87.925.

15. Li, Q.; Guan, X.; Wu, P.; Wang, X.; Zhou, L.; Tong, Y.; Ren, R.; Leung, K.S.; Lau, E.H.; Wong, J.Y. Early transmission dynamics in Wuhan, China, of novel coronavirus-infected pneumonia. New England Journal of Medicine 2020, 10.1056/NEJMoa2001316, doi:10.1056/NEJMoa2001316.

16. Tomar, A.; Gupta, N. Prediction for the spread of COVID-19 in India and effectiveness of preventive measures. Science of The Total Environment 2020, 10.1016/j.scitotenv.2020.138762, 138762, doi:10.1016/j.scitotenv.2020.138762.

17. Saez, M.; Tobias, A.; Varga, D.; Barceló, M.A. Effectiveness of the measures to flatten the epidemic curve of COVID-19. The case of Spain. Science of the Total Environment 2020, 10.1016/j.scitotenv.2020.138761, 138761, doi:10.1016/j.scitotenv.2020.138761.

18. Chakraborty, T.; Ghosh, I. Real-time forecasts and risk assessment of novel coronavirus (COVID-19) cases: A data-driven analysis. Chaos, Solitons \& Fractals 2020, 10.1016/j.chaos.2020.109850, 109850, doi:10.1016/j.chaos.2020.109850.

19. Chimmula, V.K.R.; Zhang, L. Time series forecasting of COVID-19 transmission in Canada using LSTM networks. Chaos, Solitons \& Fractals 2020, 10.1016/j.chaos.2020.109864, 109864, doi:10.1016/j.chaos.2020.109864. 
20. Melnick, H.; Darling-Hammond, L.; Leung, M.; Yun, C.; Schachner, A.; Plasencia, S.; Ondrasek, N. Reopening schools in the context of COVID-19: Health and safety guidelines from other countries. Availabe online: http://www.nordcountryschool.org/uploads/5/2/3/3/5233925/1 reopening_schools_covid19_brief.pdf (accessed on

21. Giordano, G.; Blanchini, F.; Bruno, R.; Colaneri, P.; Di Filippo, A.; Di Matteo, A.; Colaneri, M. Modelling the COVID-19 epidemic and implementation of population-wide interventions in Italy. Nature Medicine 2020, 10.1038/s41591-020-0883-7, 1-6, doi:10.1038/s41591-020-0883-7.

22. Silverman, J.D.; Hupert, N.; Washburne, A.D. Using influenza surveillance networks to estimate statespecific prevalence of SARS-CoV-2 in the United States. Science translational medicine 2020, 12, doi:10.1126/scitranslmed.abc1126.

23. Rosenberg, E.S.; Tesoriero, J.M.; Rosenthal, E.M.; Chung, R.; Barranco, M.A.; Styer, L.M.; Parker, M.M.; Leung, S.-Y.J.; Morne, J.; Greene, D. Cumulative incidence and diagnosis of SARS-CoV-2 infection in New York. medRxiv 2020, 10.1016/j.annepidem.2020.06.004, doi:10.1016/j.annepidem.2020.06.004.

24. Society, R.S. A statistician's guide to coronavirus numbers. Availabe online: https://www.statslife.org.uk/features/4474-a-statistician-s-guide-to-coronavirus-numbers (accessed on

25. Gassen, J. \{tidycovid19\}: An R Package to Download, Tidy and Visualize Covid-19 Related Data. Availabe online: https://github.com/joachim-gassen/tidycovid19 (accessed on

26. RStudio RStudio: Integrated Development for R, Version1.3.959; RStudio, PBC, : Boston, MA 2020.

27. Python Python 3 Reference Manual, Python 3.8; CreateSpace: Scotts Valley, CA, 2020.

\section{Figures}


a

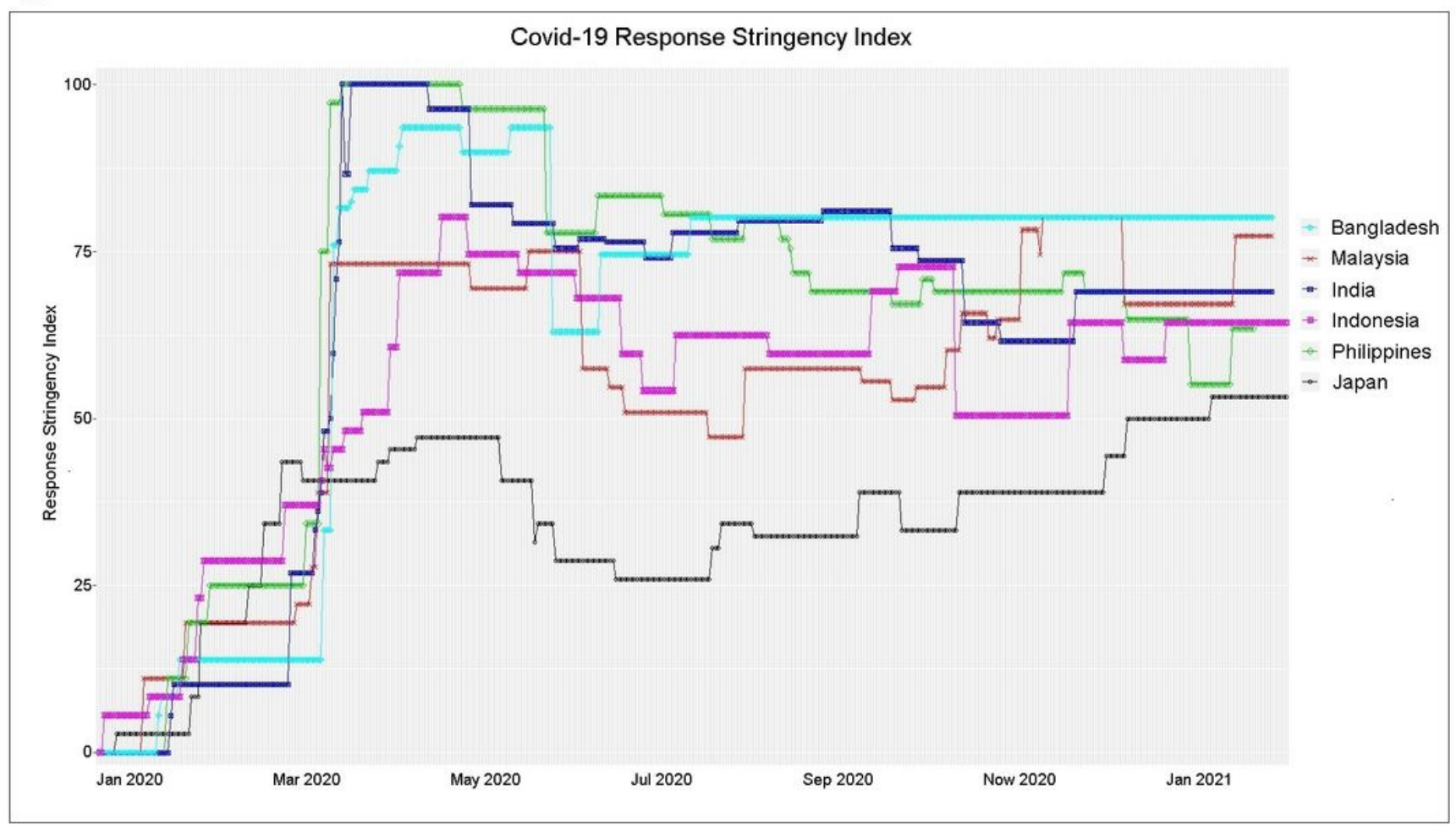

b

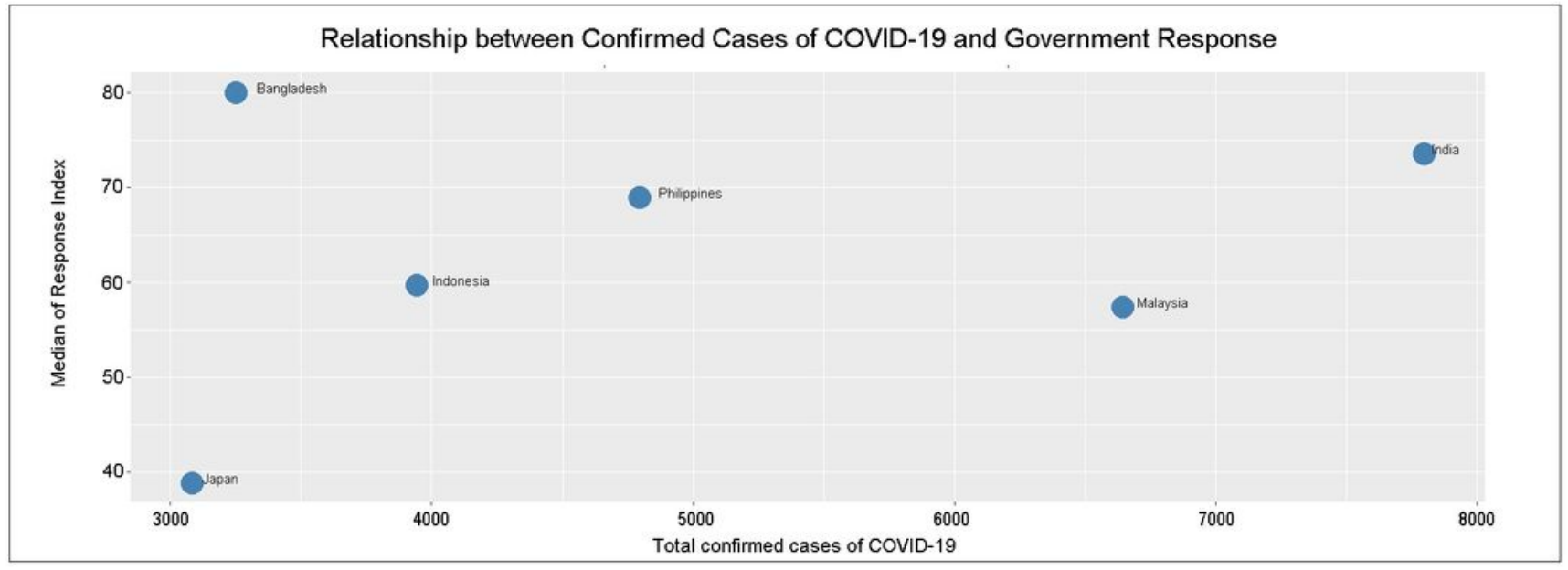

\section{Figure 1}

Interventions and responses stringency index and relationship between government response and confirmed cases. (a) Interventions and Response Stringency Index. (b) Boxplot for Interventions and Response Stringency Index. (c) Relationship between number of confirmed COVID-19 cases and government response. 
The overall situation of the epidemic in each country
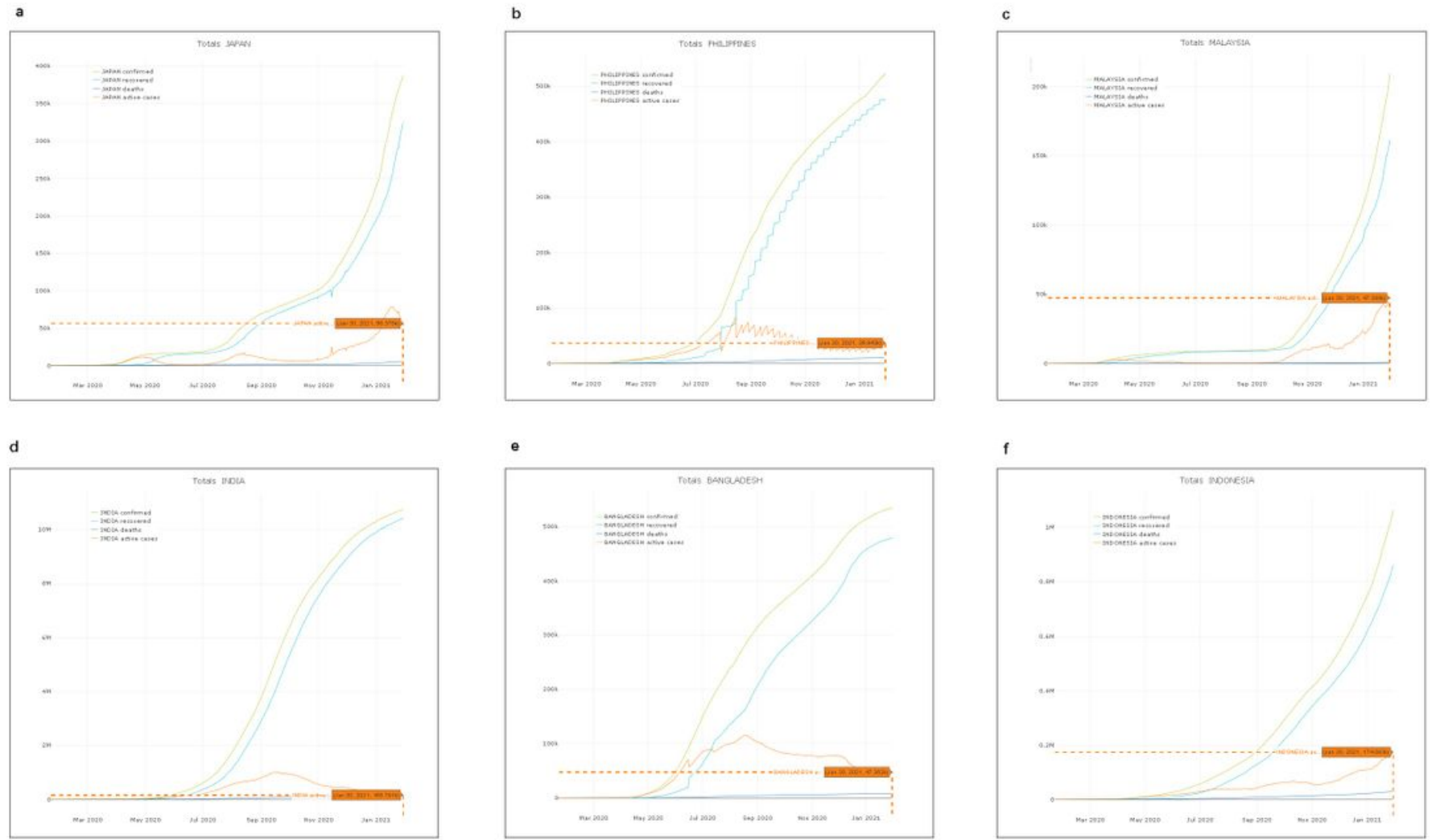

Figure 2

The overall situation of the epidemic in each country. (a-f) The overall situation of the epidemic of COVID19 in Japan, Philippines, Malaysia, India, Bangladesh and Indonesia. 

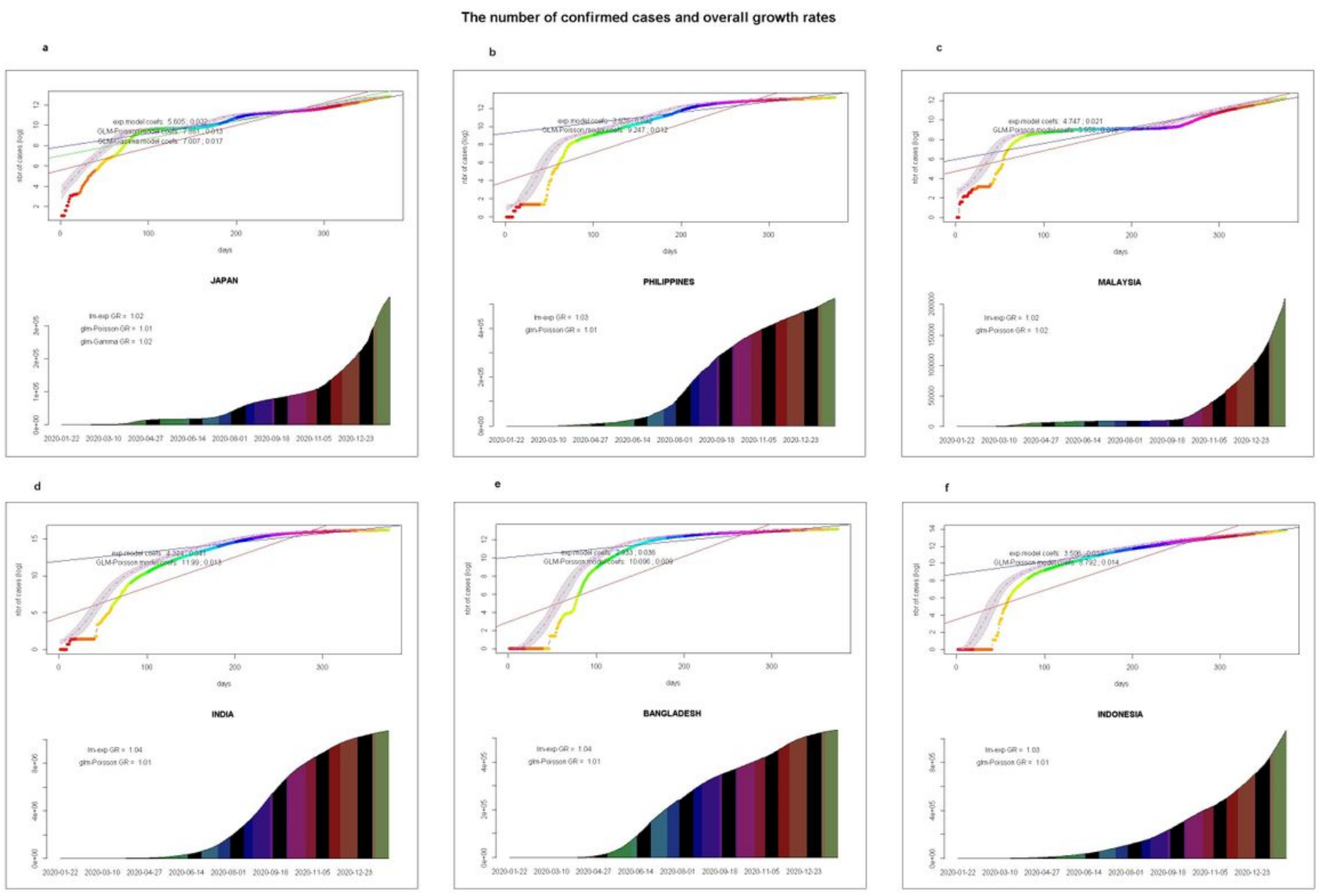

\section{Figure 3}

Total number of cases and overall growth rates for different countries. (a-f) The a-f subgraph shows Japan, Philippines, Malaysia, India, Bangladesh and Indonesia. The upper panel with a linear fit to an exponential law in log-scale and the bottom plot in linear scale in a-f subgraph. Lm-exp, an Linear Regression exponential model; glm-poisson, a General Linear Regression Poisson model; GLM-Gamma, a General Linear Regression Gamma model method. 

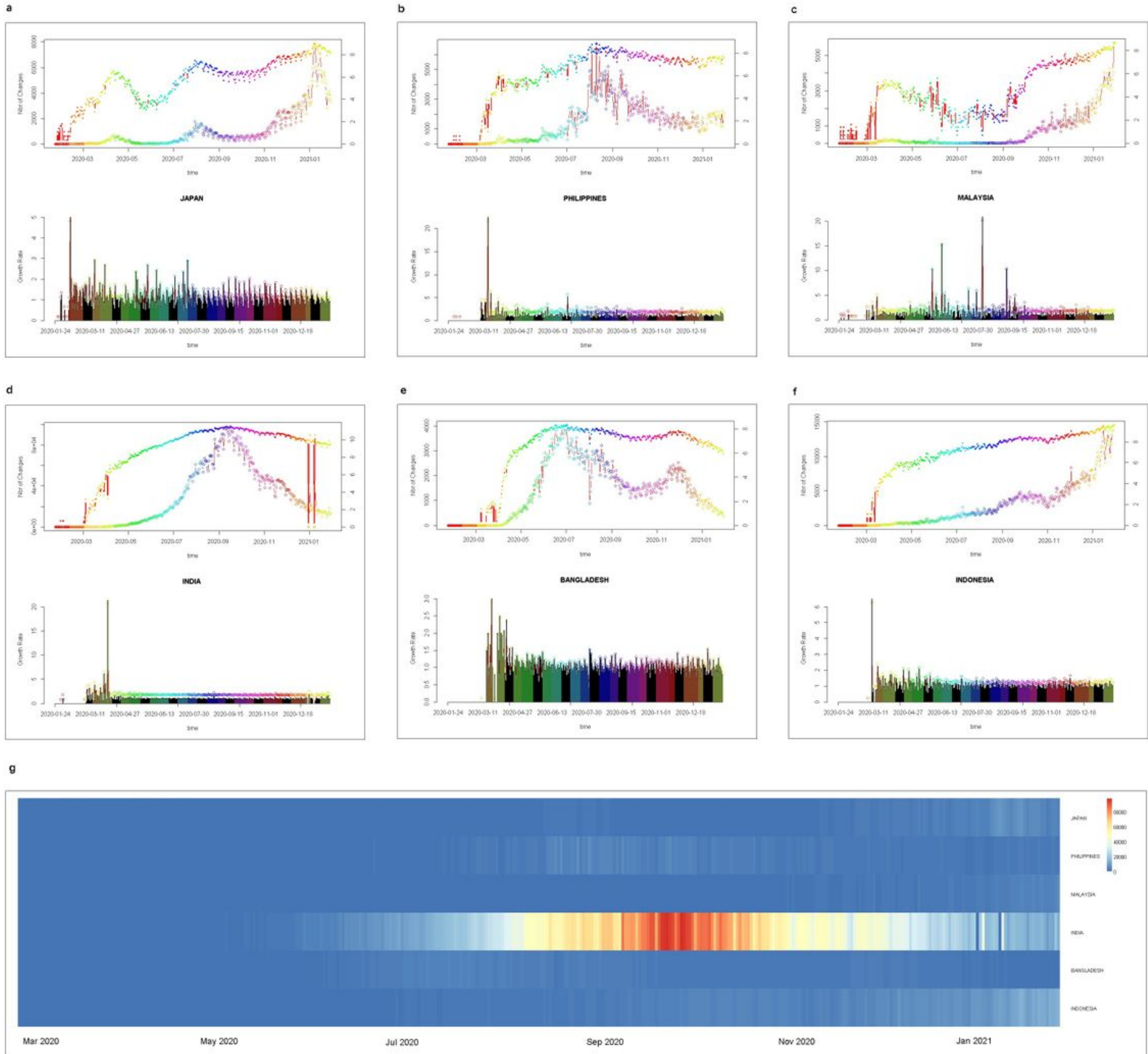

\section{Figure 4}

Estimate daily changes and growth rates per country. (a-f) The a-f subgraph shows the daily changes and growth rates in Japan, Philippines, Malaysia, India, Bangladesh and Indonesia. The upper panel, the daily number of changes basis in log scale (thicker line, right $y$-axis) and linear scale (thin line, left y-axis); the bottom panel, the daily growth rate. (g) A heatmap comparing the confirmed cases changes per day (horizontal axis) in six countries (vertical axis). 
a

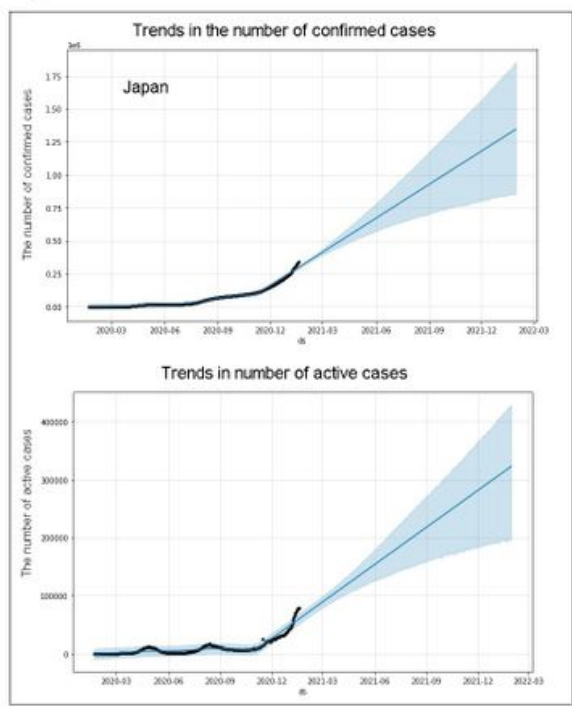

d

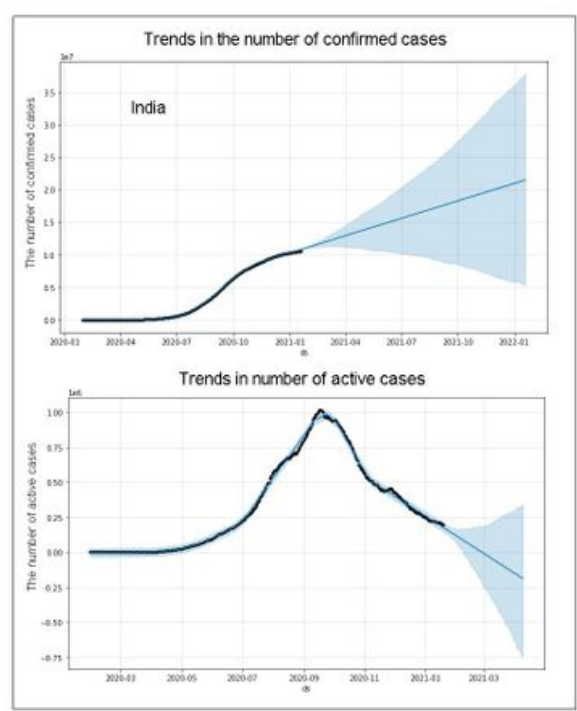

b

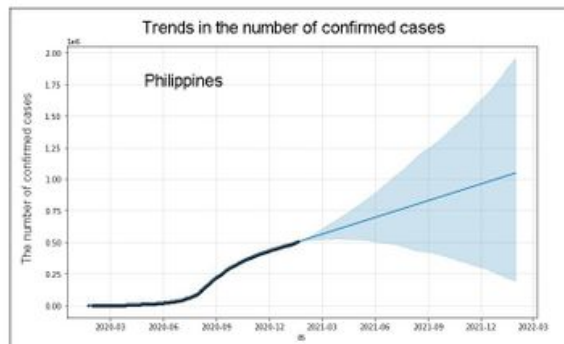

Trends in number of active cases

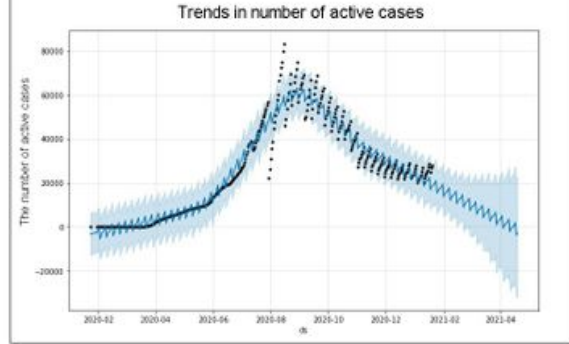

e

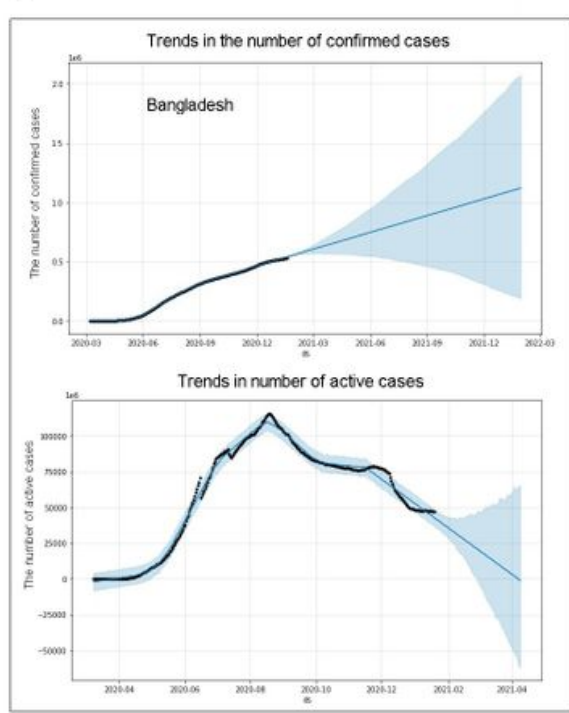

C

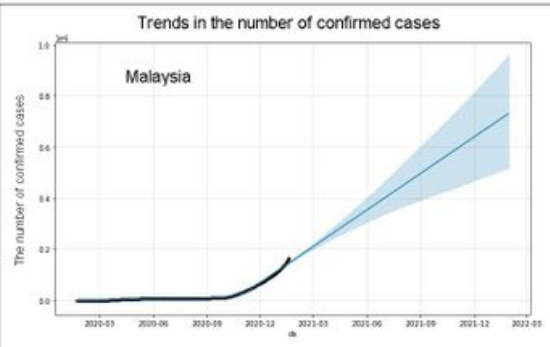

Trends in number of active cases

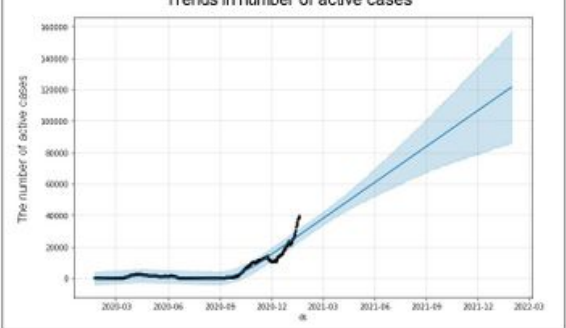

f

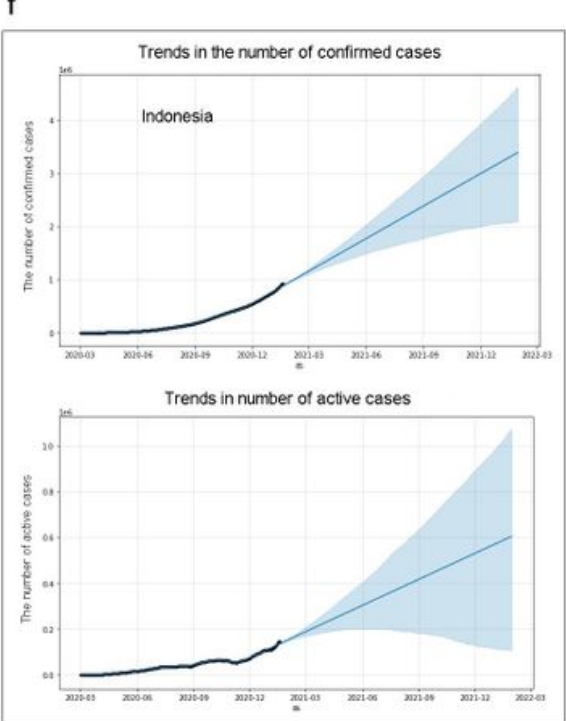

\section{Figure 5}

The trend of COVID-19 pandemic. (a-f) The trend of COVID-19 pandemic in Japan, Philippines, Malaysia, India, Bangladesh and Indonesia. The upper panel, the trend of COVID-19 pandemic in the number of total confirmed cases forecast by Machine Learning method. The bottom panel, Model and Forecast trends in number of active cases by Machine Learning method in six countries.

\section{Supplementary Files}

This is a list of supplementary files associated with this preprint. Click to download.

- FigureS1.ConfirmedcasesdeathspermillionandCFR.jpg 